\title{
UM MODELO MATEMÁTICO DO ESCOAMENTO DE LÍQUIDOS NO CADINHO DO ALTO-FORNO
}

\author{
Beatriz Fausta Gandra ' \\ José de Sousa Carvalho ${ }^{2}$ \\ José Adilson de Castro ${ }^{3}$ \\ Luiz Fernando Andrade de Castro ${ }^{4}$
}

\section{Resumo}

O cadinho, região que define a vida útil do alto-forno (AF), é onde ocorre o escoamento de gusa e escória, fenômeno que tem importante função na zona inferior do reator, afetando o seu comportamento operacional. Em função das dificuldades de se realizar medições que permitam o conhecimento do estado interno do AF, têm sido desenvolvidos modelos matemáticos que buscam simular os fenômenos que ocorrem nessa região. Neste contexto, adaptou-se um modelo de escoamento de fluidos, previamente desenvolvido pela UFF, ao AF 3 da Usiminas. Em simulações realizadas com o modelo, pode-se concluir que a determinação da distância entre o fundo do cadinho e o início da coluna de coque está intimamente ligada à variação das pressões de sopro e de topo e ao peso da carga no AF; a movimentação do líquido no cadinho é muito influenciada por essa posição do homem morto; a distribuição granulométrica do coque ao longo do raio no interior do cadinho tem forte influência nas linhas de fluxo do líquido e, conseqüentemente, na sua retenção, por outro lado, a variação homogênea do combustível não influencia de forma significativa a distribuição dos líquidos nem o tempo de residência.

Palavras-chave: Altos-fornos; Cadinho; Modelagem.

\section{A MATHEMATICAL MODEL OF LIQUID FLOW IN THE BLAST FURNACE HEARTH}

\begin{abstract}
Stable blast furnace operational is required to reduce energy consumption in iron and steelmaking industry. For the stable blast furnace operation, controlled drainage is one of the important factors. Therefore, in this work, basic characteristic features of drainage in a blast furnace hearth were examined. Three-dimensional mathematical model were developed based on the finite volume method to simulate liquid flow in a hearth of a blast furnace. Dead man position was developed to reflect the influence of same variables in it. The results indicate that the coke free space formed near the bottom of the hearth is intimately linked to the variation of the blow and top pressures and to the weight of the load in the blast furnace. Moreover, the movement of the liquid is influenced by that coke free space. In addition, the size distribution of the coke inside the hearth has strong influence in the liquid flow, and consequently, in its drainage efficiency.
\end{abstract}

Key words: Blast furnace; Hearth; Modelling.

\section{INTRODUÇÃO}

Visando uma produção econômica de ferro-gusa, via altosfornos, é importante a garantia de uma longa campanha desses reatores, aliada a uma boa estabilidade operacional. Nesse sentido, é fundamental que se tenha um cuidado especial com o cadinho, parte que define a vida útil do equipamento. Além disso, uma melhor estabilidade e eficiência da operação do forno é obtida quando se consegue uma melhor drenagem de gusa e escória, sendo para isto, necessária uma adequada permeabilidade na região do homem morto. Assim, na

\footnotetext{
'Membro da ABM; Engenheira Metalurgista, MSc.; Centro de Pesquisas e Desenvolvimento da Usiminas; Avenida Pedro Linhares Gomes, 543 I, Usiminas - 35160-900 - Ipatinga, MG, Brasil.bfgandra@usiminas.com.br

${ }^{2}$ Membro da ABM; Engenheiro Metalurgista, Superintendência de Coquerias Sinterizações e Altos-Fornos da Usiminas; Avenida Pedro Linhares Gomes, 543I, Usiminas - 35160-900 - Ipatinga, MG, Brasil. jscarvalho@usiminas.com.br ${ }^{3}$ Membro da ABM; Engenheiro Metalurgista, Dr., Universidade Federal Fluminense - UFF; Avenida dos Trabalhadores, 420 - 27255 - I25 Volta Redonda, RJ, Brasil. jose.adilson@pq.cnpq.br ${ }^{4}$ Membro da ABM, Engenheiro Metalurgista, Dr., Universidade Federal de Minas Gerais - UFMG; Escola de Engenharia, Rua Espírito Santo, 35/306, Centro - 30160-030 - Belo Horizonte, MG, Brasil. Ifac@demet.ufmg.br
} 
busca de fornecer melhores ferramentas de controle ao operador, foi desenvolvido um modelo matemático (simulação numérica) do fluxo de metal líquido no cadinho para descrever o escoamento de gusa e escória durante a corrida.

$O$ vazamento de material no AF é feito de maneira praticamente contínua, alternando-se apenas os furos de corrida. $O$ acúmulo excessivo de gusa e escória, decorrente de atraso na perfuração, má qualidade do coque, flutuações térmicas do forno e má qualidade da massa de tamponamento, provoca um aumento da pressão de sopro, descida irregular de carga, dentre outros. Além disso, as flutuações do nível de líquido podem, também, provocar um efeito prejudicial na estabilidade do reator.

Nesse contexto, o presente estudo apresenta a adaptação de um modelo matemático do fluxo de metal líquido no cadinho para ser utilizado na avaliação da movimentação do líquido no interior dos altos-fornos. As principais variáveis consideradas no modelo são: a vazão do líquido, o nível térmico do cadinho, a fração de vazios do leito de coque, a produção de gusa e a sua velocidade de movimentação no interior do cadinho.

\section{O MODELO MATEMÁTICO}

\section{I Descrição do Modelo}

O modelo, previamente desenvolvido, é tridimensional e leva em consideração a fração de vazios no interior do cadinho. Uma vez que a existência, ou não, de uma camada isenta de sólidos afeta profundamente o fluxo do gusa no cadinho, o modelo permite avaliar o perfil de velocidades para diferentes situações de permeabilidade e de assentamento do homem morto. Nas simulações, utilizam-se como dados de entrada a descrição do estado interno do cadinho, propriedades dos materiais sólidos e líquidos, condições operacionais do $\mathrm{AF}$ e uma equação de evolução da vazão de gusa.

A forma geral da conservação utilizada no modelo matemático transiente, multidimensional e multifásico é dada pela Equação I. O índice "i" representa a fase a ser considerada na solução. O coeficiente de transferência $\left(\Gamma_{\phi}\right)$ representa o produto da difusividade pela massa específica, e assume significado diferente para cada variável dependente. $\mathrm{O}$ termo fonte considera a transferência de momento entre o sólido e o líquido, que é dada pela Equação 2, enquanto a transferência de energia, para relacionar a movimentação dos líquidos com suas temperaturas, é apresentada pela Equação 3 .

$$
\begin{gathered}
\frac{\partial\left(\varepsilon \rho_{\mathrm{i}} \phi_{\mathrm{i}}\right)}{\partial \mathrm{t}}+\operatorname{div}\left(\varepsilon \rho_{\mathrm{i}} \mathrm{u}_{\mathrm{i}} \phi_{\mathrm{i}}-\varepsilon \Gamma_{\varphi_{\mathrm{i}}} \operatorname{grad} \phi_{\mathrm{i}}\right)=\mathrm{S}_{\phi \mathrm{i}} \\
\overrightarrow{\mathrm{F}_{\mathrm{s}}^{\mathrm{i}}}=\frac{\rho_{\mathrm{i}}}{\mathrm{r}_{\mathrm{h}}}\left|\overrightarrow{\mathrm{U}}_{\mathrm{i}}-\overrightarrow{\mathrm{U}}_{\mathrm{s}}\right|\left(5 \beta+0.4 \beta^{0.1}\right)\left(\overrightarrow{\mathrm{U}}_{\mathrm{i}}-\overrightarrow{\mathrm{U}}_{\mathrm{s}}\right) \\
\text { sendo, } \beta=\frac{\mu_{\mathrm{i}}}{\rho_{\mathrm{i}}\left|\overrightarrow{\mathrm{U}}_{\mathrm{i}}-\overrightarrow{\mathrm{U}}_{\mathrm{s}}\right| \mathrm{r}_{\mathrm{h}}} ; \mathrm{r}_{\mathrm{h}}=\frac{\varepsilon \phi_{\mathrm{s}} \mathrm{d}_{\mathrm{s}}}{6 \varepsilon_{\mathrm{s}}}
\end{gathered}
$$

$$
\dot{\mathrm{Q}}=\mathrm{h}_{\mathrm{s}-\mathrm{i}} \mathrm{A}_{\mathrm{si}}\left(\mathrm{T}_{\mathrm{s}}-\mathrm{T}_{1}\right)
$$

em que,

$$
\begin{aligned}
& \frac{1}{\mathrm{~h}_{\mathrm{s}-\mathrm{i}}}=\frac{1}{\mathrm{~h}_{\mathrm{s}}}+\frac{1}{\mathrm{~h}_{\mathrm{i}}} ; \quad \mathrm{A}_{\mathrm{si}}=\sum_{\mathrm{m}} \mathrm{f}_{\mathrm{m}} \frac{6 \varepsilon_{\mathrm{m}}}{\mathrm{d}_{\mathrm{m}} \phi_{\mathrm{m}}} \\
& \mathrm{h}_{\mathrm{s}}=2 \sqrt{\frac{\mathrm{k}_{\mathrm{s}} \mathrm{Cp}_{\mathrm{s}} \rho_{\mathrm{s}}\left|\overrightarrow{\mathrm{U}}_{\mathrm{i}}-\overrightarrow{\mathrm{U}}_{\mathrm{s}}\right|}{\pi \mathrm{d}_{\mathrm{s}}}} \\
& \mathrm{h}_{\mathrm{i}}=\frac{\mathrm{k}_{\mathrm{i}}}{\mathrm{d}_{\mathrm{s}}}\left[\frac{2 \sqrt{\operatorname{Re}_{\mathrm{s}-\mathrm{i}} \mathrm{Pr}_{\mathrm{i}}}}{1.55 \sqrt{\mathrm{Pr}_{\mathrm{i}}}+3.09 \sqrt{0.372-0.15 \mathrm{Pr}_{\mathrm{i}}}}\right]
\end{aligned}
$$

O cadinho pode ser representado por uma malha numérica do tipo body fitted coordinate (BFC), sendo as equações diferenciais parciais discretizadas utilizando-se o método de volumes finitos. Resumidamente, destacam-se as seguintes características para solução do modelo:(') i) uso de componente de velocidade covariante em diferentes posições; ii) uso do algoritmo SIMPLE ${ }^{(1)}$ (Semi-Implicit Method for Pressure Linked Equations) acoplando pressão e velocidade; iii) método completamente implícito para discretização no tempo; iv) TDMA (Tri-diagonal Matrix Algorithm), utilizando o método ADI (Alternate Direction Implicit), que converte o sistema de equações para um sistema tridiagonal, combinado com o método linha por linha.

Segundo a literatura, ${ }^{(2,3)}$ a saída do fluido está bastante relacionada às condições do furo de gusa. Assim, para se determinar a velocidade de saída, foram assumidas algumas considerações. $O$ fator de fricção foi determinado segundo a Equação 4 . A Equação 5 expressa a velocidade do líquido, e, multiplicada pela área do furo de gusa, leva a equação da vazão de saída do líquido (Equação 6). Além da altura de líquido no cadinho, o diâmetro do furo de gusa varia com o tempo (Equação 7).

$$
\mathrm{f}=\frac{0,0626}{\left[\log \left(\frac{\mathrm{e}}{3,7 \mathrm{D}}+\frac{5,74}{\mathrm{Re}^{0,9}}\right)\right]^{2}}
$$

$$
u=\sqrt{\left[\frac{2\left(g h+\frac{P}{\rho}\right)}{1+2 f \frac{L}{D}}\right]}
$$

$$
\mathrm{V}=\frac{\pi \mathrm{D}^{2}}{4} \sqrt{\left[\frac{2\left(\mathrm{gh}+\frac{\mathrm{P}}{\rho}\right)}{1+2 \mathrm{f} \frac{\mathrm{L}}{\mathrm{D}}}\right]}
$$




$$
\mathrm{D}=\mathrm{D}_{\mathrm{i}}+\left(\mathrm{D}_{\mathrm{f}}-\mathrm{D}_{\mathrm{i}}\right) \times\left[1-\exp \left(-1,2 \times \frac{\mathrm{t}}{\mathrm{t}_{\mathrm{R}}}\right)\right]
$$

O valor "I,2" usado na Equação 7 foi estimado a partir do perfil de desgaste de massa refratária encontrado na literatura. ${ }^{(3)}$

Baseado então nas variáveis de entrada e nas equações apresentadas, o modelo calcula a temperatura, a velocidade, a pressão e o tempo de residência do material.

\subsection{Condições de Cálculo}

As condições utilizadas nos cálculos são destacadas na Tabela I, sendo que a seguinte situação foi imposta como condição de contorno:

- a superfície do gusa na entrada, com temperatura igual à da saída, é plana e horizontal, e se mantém num nível constante;

- a velocidade de entrada do líquido é uniforme, relacionada ao ritmo de produção;

- não há transferência de massa através das paredes refratárias, sendo considerada condição de não deslizamento na face quente;

- equações de entalpia e de velocidade foram utilizadas para definir condições de contorno do fluxo na saída;

- foi especificada a temperatura inicial na face fria do refratário e a sua face superior foi considerada adiabática; e

- a flutuação do homem morto é considerada como sendo a altura calculada por um balanço de forças, descrito no ítem 2.4, para cada corrida.

Tabela I. Condição padrão para cálculo no modelo

\begin{tabular}{lcc}
\hline \multicolumn{1}{c}{ Variável } & Unidade & Valor \\
\hline Temperatura do gusa & ${ }^{\circ} \mathrm{C}$ & $15 \mathrm{I0}$ \\
Densidade do gusa & $\mathrm{g} / \mathrm{cm}^{3}$ & 6,7 \\
Rugosidade do furo de gusa & $\mathrm{mm}$ & 0,02 \\
Diâmetro inicial do furo de gusa & $\mathrm{mm}$ & $55 / 55 / 55$ \\
Comprimento do furo de gusa & $\mathrm{mm}$ & $3400 / 3200 / 3000$ \\
Pressão de sopro & $\mathrm{Pa}$ & $3,50 * 10^{5}$ \\
Pressão de saída do material & $\mathrm{Pa}$ & $1,00 * 10^{5}$ \\
Malha & $(\#)$ & $60(\mathrm{r}) \times 20(\mathrm{z}) \times 20(\theta)$ \\
Diâmetro de coque no cadinho & $\mathrm{mm}$ & 30 \\
\hline
\end{tabular}

\subsection{Validação do Modelo} (Tabela 2).

A vazão média de saída foi considerada para validação

Tabela 2. Vazão de saída do material no furo de gusa

\begin{tabular}{lccc}
\hline \multicolumn{1}{c}{ FG } & Valor modelo $(\mathbf{k g} / \mathbf{s})$ & Valor real $(\mathbf{k g} / \mathbf{s})$ & Diferença $(\%)$ \\
\hline FGI & 96,64 & 99,34 & 2,72 \\
FG3 & 99,58 & 102,44 & 2,79 \\
FG2 & 96,57 & 99,20 & 2,65 \\
Média & 97,60 & 100,33 & 2,72 \\
\hline
\end{tabular}

\subsection{Descrição do Modelo de Flutuação do Homem Morto}

Sabe-se que a distância entre o fundo do cadinho e o início da coluna de coque pode sofrer alterações durante o processo devido à quantidade de material líquido dentro do cadinho e, conseqüentemente, à força de empuxo, além das demais variáveis que atuam no núcleo do AF. Assim, baseado no balanço de forças atuando no homem morto, foi realizado, em Excel ${ }^{\circledR}$, um modelo simplificado que determina a flutuação, ou não, do homem morto, em função das condições operacionais do forno no início de cada corrida (Tabela 3).

Tabela 3. Balanço de forças para determinação da flutuação do homem morto

\begin{tabular}{|c|c|}
\hline $\begin{array}{c}\text { Forças atuando } \\
\text { para baixo }\end{array}$ & $\begin{array}{l}\text { Forças atuando } \\
\text { para cima }\end{array}$ \\
\hline $\begin{array}{l}\text { Peso do material sólido } \\
\text { Força = massa da carga }{ }^{*} \\
\text { gravidade }\end{array}$ & $\begin{array}{l}\text { Pressão do ar injetado } \\
\text { pelas ventaneiras } \\
\mathrm{F}=\text { pressão de injeção * } \\
\text { área no ventre }\end{array}$ \\
\hline $\begin{array}{l}\text { Contra-pressão no topo } \\
\text { Força = contrapressão * } \\
\text { área da goela }\end{array}$ & $\begin{array}{l}\text { Força de empuxo do } \\
\text { líquido } \\
\text { (influenciada pelo nível de } \\
\text { esgotamento) } \\
\mathrm{F}=\text { densidade do gusa * } \\
\text { volume deslocado * } \mathrm{g}\end{array}$ \\
\hline \multicolumn{2}{|c|}{$\begin{array}{l}\text { Volume ocupado pelo homem morto } \\
\text { (influenciado pela dimensão do raceway) } \\
\mathrm{F}=\mathrm{m}^{*} \mathrm{~g} \\
\mathrm{~m}=\text { densidade do coque } * \mathrm{~V} \\
\mathrm{~V}=\text { área do cadinho } * \mathrm{H} \\
\mathrm{H}=\text { altura total (ventaneira até fundo) } \\
\mathrm{x}=\text { flutuação do homem morto } \\
\mathrm{F}=\text { densidade do coque } \\
\left(\left[\mathrm{D}_{\text {cadinho }}-2 * \mathrm{D}_{\text {raceway }}\right] \wedge 2\right) / 4 * \pi *[\mathrm{H}-\mathrm{x}] * \mathrm{~g}\end{array}$} \\
\hline
\end{tabular}

\section{RESULTADOS DE SIMULAÇÕES UTIZANDO OS MODELOS}

\section{I Flutuação do Homem Morto}

Utilizando o modelo do balanço de forças apresentado pode-se avaliar a influência do comprimento do raceway, da massa da carga, do esgotamento, da pressão de injeção do ar e da contrapressão na flutuação do homem morto, considerando uma mesma produtividade do $\mathrm{AF}$ (Figura I).

Por meio da Figura I, é possível observar que a variável que mais influencia na flutuação do homem morto é a pressão de injeção do ar, seguida do peso da carga. Esse fato reforça a teoria de que paradas emergenciais no AF (variação brusca 


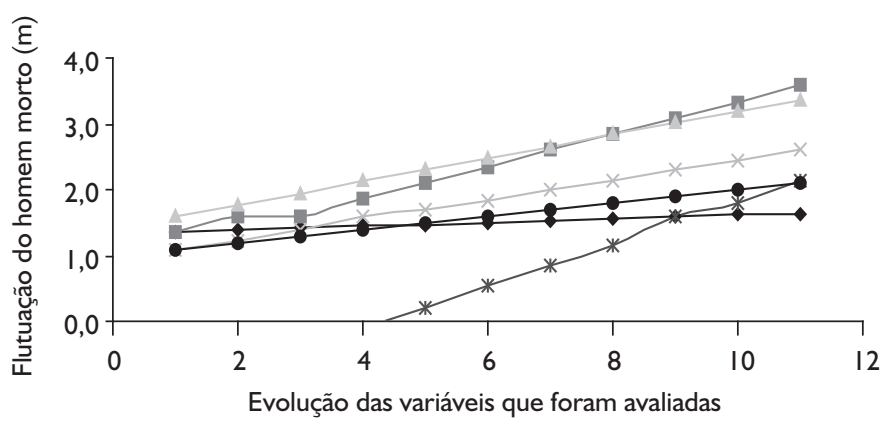

$$
\begin{aligned}
& \rightarrow \text { Comprimento do raceway }(\mathrm{I} \mathrm{m} \text { a } 2 \mathrm{~m}) \\
& \rightarrow \text { Esgotamento }(0 \mathrm{t} \text { a } 400 \mathrm{t}) \\
& * \text { Pressão do ar }\left(3,0 \mathrm{kgf} / \mathrm{cm}^{2} \text { a } 3,6 \mathrm{kgf} / \mathrm{cm}^{2}\right) \\
& - \text { Massa da carga }(\mathrm{I} 16,2 \mathrm{t} \text { a } 100,0 \mathrm{t}) \\
& * \mathrm{~N}^{\circ} \text { de cargas }(32 \text { a } 29) \\
& \rightarrow \text { Pressão do topo }\left(2,2 \mathrm{kgf} / \mathrm{cm}^{2} \text { a I,8 } \mathrm{kgf} / \mathrm{cm}^{2}\right)
\end{aligned}
$$

Figura I. Influência das variáveis no início da coluna de coque estagnado.

da pressão de sopro) devem ser evitadas ao máximo, pois causam alteração extremamente significativa na distância entre o fundo do cadinho e o início da coluna de coque, e conseqüentemente em toda movimentação do fluido no interior do cadinho. Além disso, o comprimento do raceway não apresentou influência considerável na flutuação do homem morto.

\subsection{Fluxo de Líquidos no Cadinho}

Considerando uma condição operacional padrão para o $A F$, determinou-se que a altura de flutuação do homem morto é de I,59 m. Este valor foi utilizado para alimentar o modelo do fluxo de líquidos. A evolução do tempo de residência do mate- rial líquido no cadinho foi obtida numa simulação realizada considerando-se o ciclo de três corridas consecutivas, áreas I, 3 e 2, respectivamente (Figura 2). Por esta figura, pode-se observar uma tendência de acúmulo de material na região onde não se tem vazamento, no caso, no ângulo de $0^{\circ}$. Também se observa uma saída mais rápida do material que chega sobre o furo de corrida que está em vazamento, independente de qual seja esse furo.

Escolhendo o tempo de 120 min e FGI, que foi um período de estabilização da velocidade, pode-se observar a variação das linhas de fluxo e do tempo de residência do material, para diferentes valores de diâmetro do coque (Figura 3). Não se observa influência notável da granulometria do coque no tempo de residência, nem nas linhas de fluxo, desde que o homem morto esteja sempre flutuando e a distribuição do coque seja homogênea.

Entretanto, quando se considera a variação da granulometria do coque ao longo do raio, fica visível a influência desse combustível nas linhas de fluxo, e por conseqüência no tempo de residência do líquido (Figura 4).

Também em conformidade com a literatura, pode-se observar uma grande influência da situação do homem morto na velocidade do material e no seu tempo de residência (Figura 5).

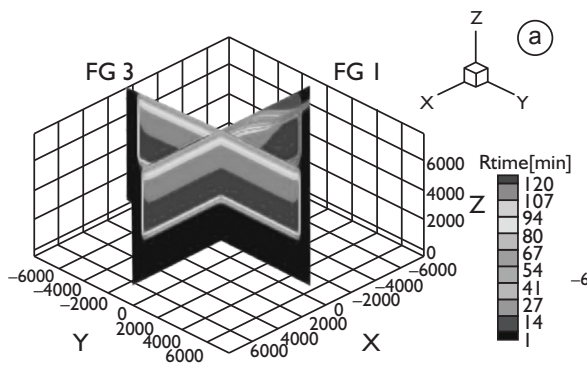

FG 2

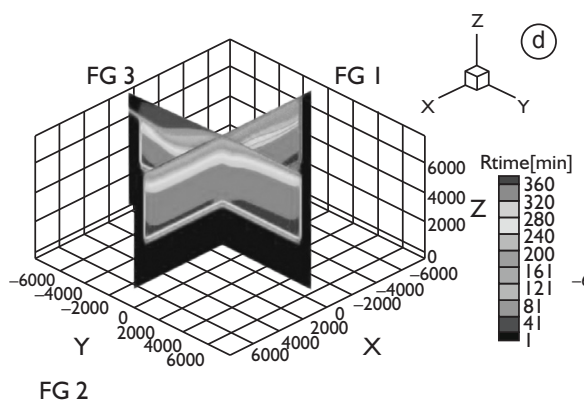

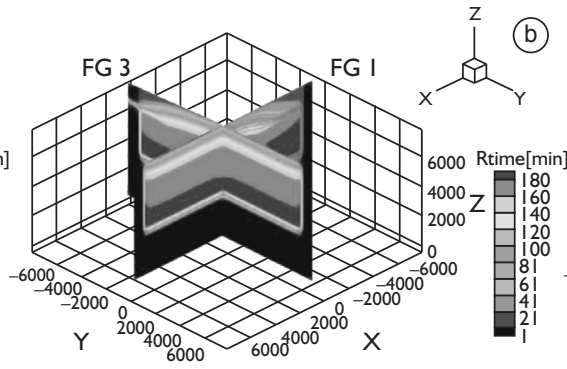

(b)

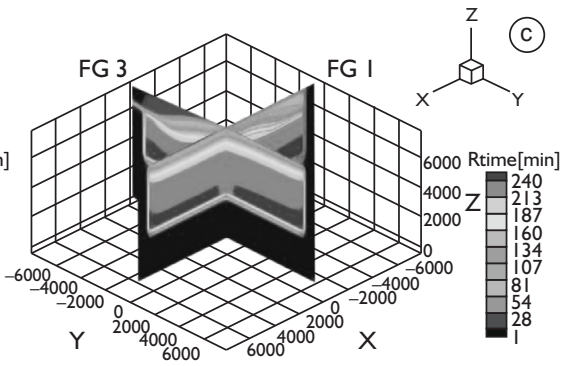

FG 2

Figura 2. Evolução do tempo de residência do material líquido. a) $t=(20 \mathrm{~min} ; b) t=(80 \mathrm{~min} ; c) t=240 \mathrm{~min} ; \mathrm{d}) \mathrm{t}=360 \mathrm{~min} ; \mathrm{e}) \mathrm{t}=410 \mathrm{~min}$; f) $\mathrm{t}=470 \mathrm{~min}$. 

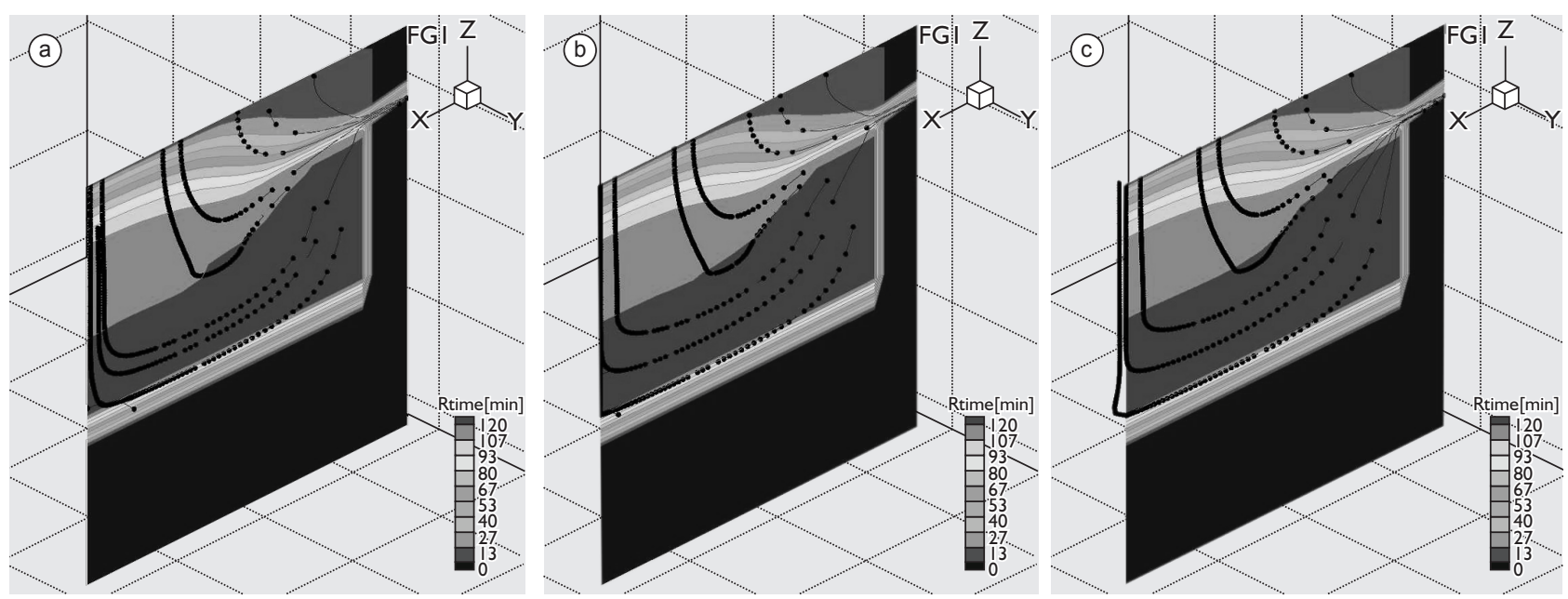

Figura 3. Linha de fluxo e tempo de residência para diferentes valores de diâmetro do coque. a) $d_{\text {coque }}=15 \mathrm{~mm}$; b) $d_{\text {coque }}($ padrão) $=30 \mathrm{~mm}$; c) $\mathrm{d}_{\text {coque }}=60 \mathrm{~mm}$.
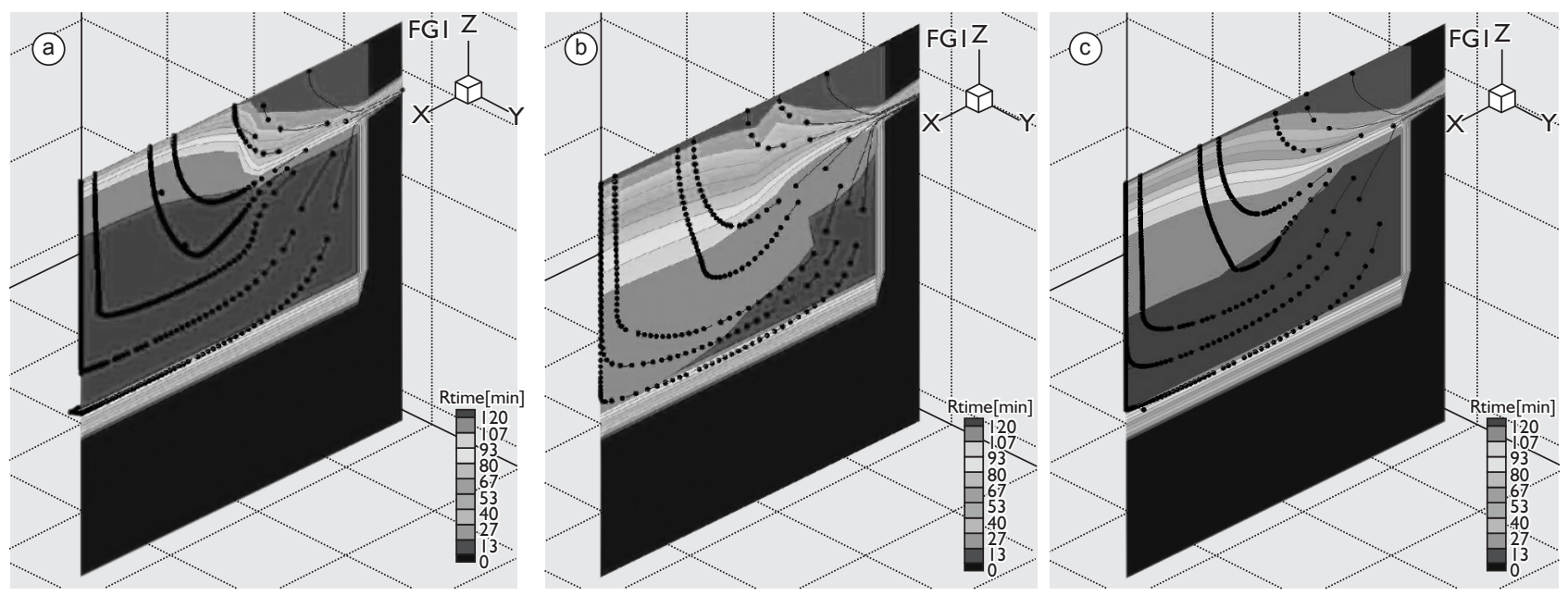

Figura 4. Linhas de fluxo, ao avaliar a distribuição do coque no cadinho. a) $d_{\text {coque }}=30 \mathrm{~mm}$ e $60 \mathrm{~mm}$; b) $d_{\text {coque }}=60 \mathrm{~mm}$ e I5 mm; c) $\mathrm{d}_{\text {coque }}=30 \mathrm{~mm}$ (padrão).
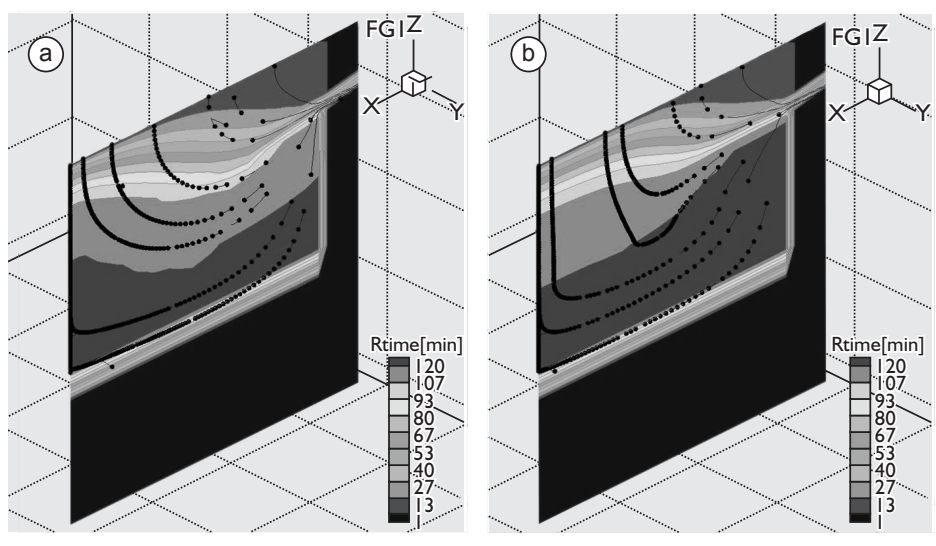

Figura 5. Linhas de fluxo, avaliando a alteração do homem morto. a) homem morto assentado; b) homem morto flutuando.

\section{CONCLUSÕES}

Neste trabalho foi realizado um estudo sobre a movimentação do líquido no interior do cadinho do AF. Este estudo englobou o desenvolvimento de um balanço de forças que permite avaliar a flutuação do homem morto no cadinho, baseado nas características do reator e em suas condições operacionais. O resultado foi então utilizado para alimentar o modelo matemático previamente desenvolvido. Além disso, foi realizada a implementação, neste modelo, de uma nova rotina para cálculo da velocidade de saída do material, considerando a variação da altura de líquidos e do diâmetro do furo de gusa com o 
tempo, e uma rotina que permite alterar a distribuição da granulometria do combustível ao longo do raio do cadinho. $O$ modelo se mostrou consistente e os resultados alcançados permitiram concluir que: i) a determinação da distância entre o fundo do cadinho e o início da coluna de coque está intimamente ligada à variação de pressão de sopro e de topo e ao peso da carga no reator; ii) a movimentação do líquido no cadinho é muito influenciada pela situação de flutuação, ou não, do homem morto. Quando o homem morto se apresenta assentado no cadinho, o fluido tende a ir diretamente para o furo de gusa. Por outro lado, quando o homem morto está flutuando, o líquido primeiramente vai para a região sem coque e, posteriormente, segue para o furo de gusa; iii) a variação homogênea da granulometria do coque não influenciou de forma significativa a distribuição dos líquidos nem o tempo de residência. No entanto, a distribuição granulométrica do coque ao longo do raio no interior do cadinho tem forte influência nas linhas de fluxo do líquido, e conseqüentemente, na sua retenção.

\section{REFERÊNCIAS}

I PATANKAR, S.V. Numerical heat transfer and fluid flow. Washington, DC: Hemisphere/Taylor \& Francis, 1980.

2 GANDRA, B.F.; SCUDELLER, L.A.M.; CASTRO, J.A.; CASTRO, L.F.A. Fluxo de líquidos no cadinho do alto-forno. In: SEMINÁRIO DE REDUÇÃO DE MINÉRIO DE FERRO E MATÉRIAS-PRIMAS, 35., 2005, Florianópolis, SC. Anais... São Paulo: ABM, 2005. I CD-ROM. p. 236-45

3 GANDRA, B.F. Desenvolvimento de modelo matemático do fluxo de líquidos no cadinho do alto-forno. 2007. 86 p. [Dissertação de Mestrado em Engenharia Metalúrgica e de Minas] - Curso de Pós-Graduação em Engenharia Metalúrgica e Materiais da Universidade Federal de Minas Gerais, Belo Horizonte, 2006.

Recebido em: 07/05/08

Aceito em: 02/12/08

Proveniente de: SEMINÁRIO DE REDUÇÃO DE MINÉRIO DE FERRO E MATÉRIAS-PRIMAS, 37., 2007, Salvador, BA. São Paulo: ABM, 2007.

\section{SÍMBOLOS E ABREVIATURAS}
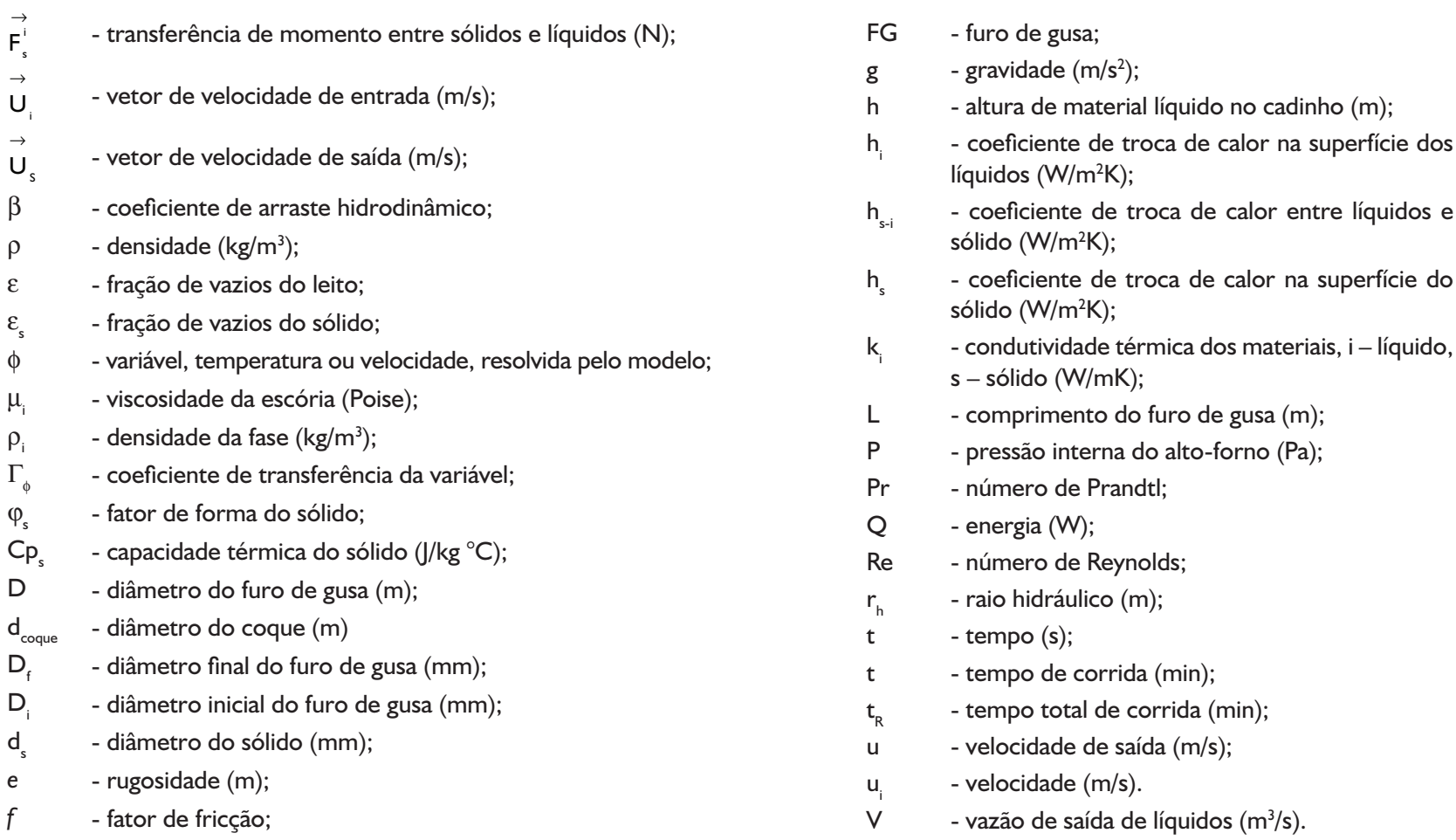